\title{
SURFACE ACTIVATION LEUKOCYTE MARKERS AND HUMORAL FACTORS IN CORD BLOOD OF NEWBORNS AT RISK OF EARLY INFECTION
}

\author{
Pavel Rozsíval', Eva Pařizková1, Doris Vokurková ${ }^{2}$ Božena Buriánková1, Ctirad Andrýs ${ }^{2}$
}

Charles University in Prague, Faculty of Medicine in Hradec Králové and University Hospital Hradec Králové, Czech Republic: Pediatric Department ${ }^{1}$, Institute of Clinical Immunology and Allergology ${ }^{2}$

Summary: Due to immaturity of both specific and non-specific immune mechanisms, neonates are at risk of serious infections. The risk group definition is vague, clinical signs are non-specific and common laboratory markers are not as useful as in later ages, especially due to delayed reactions. In an attempt to find early, sensitive and specific markers, we assessed a defined set of surface leukocyte markers and humoral factors in cord blood. Several differences were noted - children in the risk group had a higher proportion of CD19+/23+, CD16+/64+, CD45RO cells and higher levels of IL-6. We find it promising that already at birth there are notable signs of reaction to infection and that a follow-up of a set of infectious markers could be useful to identify the children in need of antibiotic treatment and for diminishing unnecessary treatment.

Key words: Newborns; Early infection; Leukocyte surface markers; Interleukin; Neopterin; Procalcitonin

\section{Introduction}

Due to the immaturity of their immune system, both of the non-specific and specific mechanisms, newborns are at risk of infection. Generalization of infection occurs easily, leading to overwhelming sepsis. Part of the infections is acquired in utero (infection presents an important cause of premature birth), part during the passage through the colonized birth canal and part shortly after the birth. "Early" and "late" infections are discriminated because of different causative agents and thus need different treatment. The arbitrary threshold for early infection commonly used in the Czech Republic is $\leq 72 \mathrm{~h}$ at the first sign of the disease, although most of the "early" infections manifest earlier. The largest proportion of the early infections are bacterial, E. coli and Group B Streptococcus (GBS, e.g. S. agalactiae) are the two most common causative microbes. The neonatal immune system, although capable of reaction comparable to adults (1), is naive and mounting full reaction takes more time than later in life. Common infectious signs (fever, tachycardia, dyspnea, jaundice, petechiae, exanthema...) are non-specific in neonates, as there are many non-infectious causes, and the rise of common laboratory markers (e.g. CRP) can be delayed up to $48 \mathrm{~h}$ due to the slow immune reactivity. The presence of the acknowledged risk factors leading to preventive antibiotic treatment occurs in many neonates who will subsequently not develop early infection; conversely, any risk factors may not be noted before the early infection develops. With a wide set of surface leukocyte activation markers and humoral factors, we tried to find differences between cord blood of the neonates at risk and not at risk of infection, which could help in decisions about the antibiotic treatment.

\section{Materials and methods}

\section{Materials}

Samples of cord blood for flow cytometry (approx. 0.5 $\mathrm{ml}$ ) and for humoral factor measurements (approx. 3-4 ml) from 82 patients were gathered through 2003 and 2004, consecutively from as many risk neonates as possible. All parents were given written information and signed informed consent that was approved by the University Hospital's Ethical Committee. Fetal and maternal risks were defined; fetal being prematurity, hypotrophy, hypertrophy, clinical signs of infection at birth; maternal being premature rupture of membranes (PROM), GBS positivity/other pathologic colonization or unknown status, febrile illness, CRP elevation > $10 \mathrm{mg} / \mathrm{l}$, diabetes mellitus. Presence of one or more risk factors classified the child into the risk group. 55 children were placed into the risk group, 27 served as controls.

\section{Methods}

Flow cytometry analysis

Leukocyte subpopulations were measured by two- or three-color flow cytometry with Epics XL (Beckman Coulter). Monoclonal antibodies against CD4, CD8, CD19, 
CD16 and isotype control IgG1 were purchased from Immunotech (France). Monoclonal antibodies against CD69, CD45-RA, CD45/RO, CD25, CD57, CD38, CD23, CD14, HLA-DR, CD64 and CD11b were from DakoCytomation (Denmark). The antibodies were marked with one of three fluorochromes: fluorescein isothiocyanate (FITC), phycoerythrin (PE) and phycoerythrin-Cyanin5 (PC5). The cells were identified by combinations as follows: CD69 (FITC)/CD25 (PE)/CD3 (PC5) - activated T lymphocytes, CD69 (FITC)/CD23 (PE)/CD19 (PC5) activated B lymphocytes, CD45-RO (PE)/CD45-RA (PE)/CD4 (PC5) - naive and memory T helper lymphocytes, CD57 (FITC)/CD38 (PE)/CD8 (PerCP) - NK cells and activated cytotoxic $\mathrm{T}$ cells. Monocyte population was measured by combinations CD14 (FITC)/HLA-DR (PE)/CD16 (PC5) and granulocytes were determined by CD64 (FITC)/CD11b (PE)/CD16 (PC5). A sample of 10 $\mu \mathrm{l}$ of each monoclonal reagent triple was added to $50 \mu \mathrm{l}$ citrated umbilical cord blood in $12 \times 75$ test tubes, centrifuged gently and incubated at room temperature for $15 \mathrm{~min}$ in the dark. Red blood cells were lysed using $250 \mu \mathrm{l}$ of Optilyse@ C lysing solution (Immunotech, Beckman Coulter), containing $<5 \%$ formaldehyde and incubated for $15 \mathrm{~min}$ at room temperature in the dark. The cells were resuspended with phosphate-buffered saline and analyzed immediately.

Flow cytometric analysis was performed on a Coulter Epics XL flow cytometer equipped with a $15 \mathrm{~mW}$ argon-ion laser with excitation capabilities at $488 \mathrm{~nm}$ (Beckman Coulter). A minimum of 10000 cells was collected for each sample in a list mode file format. List mode data were analyzed using Epics XL System II software (Coulter Electronic, USA).

\section{Humoral factors measurement}

Serum concentrations of interleukins IL-12 and IL-10 were determined using commercial ELISA kits (R\&D Systems, USA). IL-6 was measured by chemiluminiscent immunoassay (DPC, USA), levels of neopterin were evaluated using ELISA technique (Brahms, Germany) and concentrations of procalcitonin were determined using chemiluminiscent immunoassay (Brahms, Germany).

\section{Statistics}

Number Cruncher Statistical Software (NCSS), parametric and non-parametric tests, was used for comparison of the results between the groups.

\section{Results}

The risk group tended to be younger and to have lower birth weight than the control group but the difference was not statistically significant. In the surface markers section (results presented as mean $\%$ of cells $\pm \mathrm{SD}$, threshold $\mathrm{p}$ value for significance set to 0.05 , power $\geq 80 \%$ was searched where possible), no differences between the two groups were noted in the basic populations of immune cells - T, T- helpers, T-cytotoxic, B and NK cells. Neonates in the risk group had significantly higher proportion of $\mathrm{CD} 3+$ / CD45RO+ memory T-cells: $2.21 \pm 1.13 \%$, in comparison to $1.67 \pm 0.90 \%$, the power of this statement however is not satisfactory. Significant difference was also reached for CD64 activation marker on granulocytes: $4.64 \pm 6.61 \%$ for risk and $2.17 \pm 2.13 \%$ for control group. The power of this statement is not over $80 \%$ either. There was a tendency for CD23+ B cells to be higher in risk patients $(31.45 \pm 10.26 \%$ risk to $26.98 \pm 9.80 \%$ control group), however, the $\mathrm{p}$ value was not significant ( 0.06). Also, CD14+/CD16+ monocytes tended to be higher in risk patients $(10.46 \pm 9.56 \%$ risk to $6.40 \pm 2.94 \%$ control group).

In the humoral factors section, only the difference in IL-6 level reached statistical significance: 7.21 \pm 3.72 $\mathrm{pmol} / 1$ for risk to $3.83 \pm 3.23 \mathrm{pmol} / 1$ for control group. Levels of IL-10, IL-12, neopterin and procalcitonin were not statistically different between the groups.

Tab. 1: Comparison of differences between risk and control group.

\begin{tabular}{|c|c|c|c|c|}
\hline \multirow{2}{*}{$\begin{array}{c}\text { Variable } \\
\text { (unit) }\end{array}$} & \multicolumn{2}{|c|}{ Risk group } & \multicolumn{2}{c|}{ Control group } \\
Mean & SD & Mean & SD \\
\hline CD3/CD45RO (\%) & 2.21 & 1.13 & 1.67 & 0.9 \\
\hline CD64/CD11b/CD16 (\%) & 4.64 & 6.61 & 2.17 & 2.13 \\
\hline CD23/CD19 (\%) & 31.45 & 10.26 & 26.98 & 9.8 \\
\hline CD14/CD16 (\%) & 10.46 & 9.56 & 6.4 & 2.94 \\
\hline IL-6 (pmol/1) & 7.21 & 3.72 & 3.83 & 3.23 \\
\hline
\end{tabular}

\section{Discussion}

Many possible markers of early infection have been investigated so far, yielding variable results, in search of a rapid, specific and sensitive test that would ensure early treatment and reduce unnecessary prophylactic treatment $(3,4,5,6)$. Many humoral factors, recently even contents of their mRNA, have been measured, attempting to discover the immune reaction as early as possible. Also, many surface markers on various cell lineages involved in the immune defense have been investigated $(8,9,11)$. Our aim was to be minimally invasive and this was fulfilled by using small amounts of cord blood. This was, however, our limitation too because we were not able to measure the investigated markers sequentially in time, which could be valuable due to variable dynamics of individual markers. Our study was unique in two ways - first, it is a wide range of surface markers combined with humoral factors measured at the same time. Second, it is the attempt to discriminate between the risk and control group by the correlation of commonly used risk factors and the laboratory values measured at the same time - at birth, when the clinician has to decide whether to initiate the prophylactic antibiotic treatment or not. It is very early in the time sequence of the development of the infection - most early infections are acquired very shortly before birth or at birth, thus giving too little time for the de- 
velopment of a notable reaction in the cord blood. This is, most probably, the reason why we didn't show any strong differences between the risk and control group. The differences shown by us coincide with previous findings from other studies (e.g. 7) and with the common knowledge of the pathophysiology of inflammatory responses. The recognized facts are useful for further study because, as concluded from previous studies, the positivity of one marker may not be proved in another study, or might be shown nonspecific, as happened with IL-6 $(2,10)$. The influence of partially different material - cord blood in our study vs. venous blood in other studies - is also possible. Thus, a combination of markers shown by us to be the most promising, covering several cell lineages, followed sequentially, should show higher sensitivity and specificity in subsequent studies.

\section{Conclusions}

There are very little differences in laboratory markers of early infection between neonates positive for the commonly used risk factors and neonates free of risk factors. A combination of markers could help increase sensitivity. In our study, the combination of CD64 on granulocytes with CD23 on B cells, CD14+ on monocytes and IL- 6 seemed promising.

\section{Acknowledgements}

This study was supported by grant no. 7533-3 from Internal Grant Agency of the Ministry of Health of Czech Republic.

\section{References}

1. Berner R, Welter P, Brandis M. Cytokine Expression of Cord and Adult Blood Mononuclear Cells in Response to Streptococcus agalactiae. Pediatr Res 2002;51:304-9.

2. Chiesa C, Pellegrini G, Panero A, Osborn JF, Signore F, Assumma M, Pacifico L. C-Reactive Protein, Interleukin-6, and Procalcitonin in the Immediate Postnatal Period: Influence of Illness Severity, Risk Status, Antenatal and Perinatal Complications, and Infection. Clin Chemistry 2003;49:60-8.

3. Dollner H, Vatten L, Austgulen R. Early diagnostic markers for neonatal sepsis: Comparing C-reactive protein, interleukin-6, soluble tumour necrosis factor receptors and soluble adhesion molecules. J Clin Epidemiol 2001;54: 1251-7.

4. Hatzidaki E, Gourgiotis D, Manoura A, Korakaki E, Bossios A, Galanakis E, Giannakopoulou C. Interleukin-6 in preterm premature rupture of membranes as an indicator of neonatal outcome. Acta Obstet Gynecol Scand 2005;84: 632-8.

5. Kayem G, Goffinet F, Batteux F, Jarreau PH, Weill B, Cabrol D. Detection of interleukin-6 in vaginal secretions of women with preterm premature rupture of membranes and its association with neonatal infection: A rapid immunochromatographic test. Am J Obstet Gynecol 2005;192:140-5.

6. Ng PC: Diagnostic markers of infection in neonates. Arch Dis Child Fetal Neonatal Ed 2004;89:229-35.

7. Skrzeczynska J, Kobylarz K, Hartwich Z, Zembala M, Pryjma J. CD14+CD16+ Monocytes in the Course of Sepsis in Neonates and Small Children: Monitoring and Functional Studies. Scand J Immunol 2002;55:629-38.

8. Tezuka T, Sugita K, Mizobe N, Goi K, Miyamoto N, Nakamura M, Kagami K, Yokoyama T, Nakazawa S. Transient increase in CD45RO expression on T lymphocytes in infected newborns. Pediatr Res 1998;43(2):283-90.

9. Weirich E, Rabin RL, Maldonado Y, Benitz W, Modler S, Herzenberg LA Herzenberg LA. Neutrophil CD11b expression as a diagnostic marker for earlyonset neonatal infection. J Pediatr 1998;132:445-51.

10. Yanowitz TD, Jordan JA, Gilmour CH, Towbin R, Bowen A, Roberts JM, Brozanski BS. Hemodynamic Disturbances in Premature Infants Born after Chorioamnionitis: Association with Cord Blood Cytokine Concentrations. Pediatr Res 2002;51:310-6.

11. Yoon BH, Romero R, Shim JY, Shim SS, Kim CJ, Jun JK. C-reactive protein in umbilical cord blood: a simple and widely available clinical method to assess the risk of amniotic fluid infection and funisitis. J Maternal-Fetal Neonatal Med $2003 ; 14: 85-90$

\section{Corresponding author:}

Pavel Rozsíval, M.D., Pediatric Department, University Hospital Hradec Králové, Sokolská 581, 50005 Hradec Králové, Czech Republic, e-mail: pavel.rozsival@seznam.cz 